\title{
Wissensstrukturierung und Wissensvermittlung durch Routineformeln: Am Beispiel ausgewählter althochdeutscher \\ Texte
}

\author{
NATALIA FILATKINA and MONIKA HANAUSKA
}

Abstract

The following paper explores the role of routine formulae in the processes of knowledge structuring and transmission in the Early Middle Ages. The term "formulaic language" is understood as involving syntactically, semantically and pragmatically more or less fixed expressions or texts which are an important part of cultural practice and communication. Although recent phraseological research has demonstrated an important role for formulaic patterns in modern European languages, historical aspects of formulaic language have not received a great deal of attention, there existing only small number of studies dealing with this topic, mainly focused on one author or one type of text such as blessings or conversational guides.

By collecting and systematically exploiting a corpus of German historical texts from 750 to 1750, the research group "Historical Formulaic Language and Traditions of Communication" at the University of Trier, Germany is able to expand knowledge of the historical functions of formulaic language. The project has developed a methodology for investigation all types of historical formulaic patterns. Its database includes all Old High German texts with the exception of glosses.

The following paper concentrates on only one type of phrasal vocabulary routine formulae and explores their use and pragmatic function in a number of vernacular Old High German texts. The study focuses on selected Old High German texts used for didactic purposes as well as biblical exegesis and biblical epics. Knowledge for this study is understood chiefly as a function of the scientific heritage of Antiquity and Christianity which was required to be made accessible to students in the medieval classroom. The paper presumes that formulaic language is a fundamental element of verbal communication in the Middle Ages and the Early Modern Period just as it is in contemporary communication. This assumption is supported by the fact that routine formulae do play a significant 
part in knowledge transmission in the Middle Ages and have many pragmatic functions in the source documents.

The selection of texts is based on the understanding that academic life in the Early Middle Ages was mainly dominated by monastic culture. In this environment, theological and philosophical knowledge was transmitted to students on the one hand and to educated scholars on the other, largely in Latin as a predominant academic language and as a connecting bridge for the scholars all over Western Europe. Vernacular languages such as Old High German did not play an important role in this context. Nevertheless, there are a small number of texts that show that Old High German was also a part of this scholarly tradition. In such texts as Williram's of Ebersberg paraphrase of the Song of the Songs, the biblical epic of Otfrid of Weißenburg or Notker's of St. Gall scholarly adaptation of classical authors and texts like Martianus Capella, Boethius or the Psalms, scholarly knowledge is transmitted to a monastic audience. In these texts, routine formulae play a significant role in knowledge transmission. The authors use formulaic patterns systematically to prepare their texts for their readers. Four main groups of routine formulae have been differentiated according to their chief pragmatic function.

The first group of routine formulae is used to direct the readers' attention to new or important contents. These expressions also introduce new content, and structure the text, e.g. fernemet/firnim ('notice, listen!'). In the second group the chief pragmatic function is to instruct the reader/listener by emphasizing important aspects of information, e.g. ih sago dir ('I may tell you'), giuuisso unizist thu thaz ('you may know') or ih uneiz unola ('I know well'). The routine formulae of the third group are mainly used to point to matters of interest, e.g. hier ist ze unizzene ('here you have to know') or hier ist ze merchene ('here you have to notice'). The function of the fourth group is to explain previous passages which are difficult to understand or to translate into German, as e.g. daz chit ('this means') or ih meino ('I mean'). As routine formulae are often multifunctional, it is not always possible to strictly separate the first three groups from each other. There are many overlaps between these groups. It is therefore necessary to take the variety of pragmatic functions into account for an adequate description of these routine formulae.

The results of this study show an abundant usage of routine formulae, the diversity of their forms and functions as well as their importance to the structure of Old High German texts. That being so, new light has been thrown on the historical significance of formulaic language, and ways of investigating historical traditions of communication have been illustrated.

Keywords: routine formulae; Old High German; knowledge transfer; formulaic patterns; pragmatic function. 


\section{Einleitung}

Der vorliegende Aufsatz widmet sich der Rolle der Routineformeln in historischen Texten der Wissensvermittlung und Wissensstrukturierung und bezieht diese Begriffe auf das gelehrte (u.a. biblische und theologische) Wissen der klösterlichen Schulausbildung im Frühen Mittelalter. Im Mittelpunkt stehen deshalb einige ausgewählte althochdeutsche ${ }^{1}$ Texte, für die die Verwendung der wissensvermittelnden Routineformeln besonders charakteristisch ist. Die Auswahl der Texte beruht auf den Ergebnissen der systematischen Auswertung der gesamten althochdeutschen Überlieferung nach dem Auftreten formelhafter Wendungen durch die Nachwuchsforschergruppe „Historische Formelhafte Sprache und Traditionen des Formulierens (HiFoS)“" an der Universität Trier. ${ }^{2}$ Das Projekt widmet sich der epochenübergreifenden Dokumentation und Untersuchung der Gebrauchsdynamik und Variation der historischen formelhaften Wendungen in unterschiedlichen Textsorten aus der Zeitspanne zwischen ca. 750 bis ca. 1750. Es wird von der Überzeugung geleitet, dass Formelhaftigkeit ein Grundkonstituens der sprachlichen Repräsentation von Weltwissen ist. Im Mittelalter und in der Frühen Neuzeit war sie ein wesentliches Element der verbalen Kommunikation und kam vor allem mit Hilfe der syntaktisch, semantisch und pragmatisch mehr oder weniger fest werdenden bzw. gewordenen Wendungen oder Texte zustande. Diese stellten eine herausragende kulturelle Kommunikationspraktik mit besonderem Status dar und gestalteten die Traditionen des Formulierens. Sie basierten einerseits auf kulturell geprägten gesellschaftlichen Gebrauchskonventionen, waren aber andererseits historischen Veränderungsprozessen unterworfen.

Für die Gegenwart haben die Korpus- und Computerlinguistik bereits in den 90er Jahren des vergangenen Jahrhunderts empirisch anhand elektronischer Textkorpora nachgewiesen, in welch hohem Maße die meisten in Europa verbreiteten Sprachen formelhaft (syntaktisch und semantisch) geprägt sind. ${ }^{3}$ $\mathrm{Zu}$ einer ähnlichen Schlussfolgerung kommen auch die Untersuchungen aus den Bereichen der Formulierungstheorie, Textsortenlinguistik und Ritualforschung. Der Hintergrundgedanke ist dabei, dass Wörter einer Sprache nicht isoliert voneinander funktionieren, sondern syntagmatisch und eben phraseologisch miteinander verbunden sind und nur in dieser Verbundenheit in verschiedenen Kommunikationssituationen Sinn ergeben. Viele der in der Kommunikation auszuführenden Sprachhandlungen sind konventionalisierte, ritualisierte Kommunikationsformen, die das Formulieren ökonomischer gestalten. Die Konventionalisierung, Routine in der Mündlichkeit (Coulmas 1981) wie in der Schriftlichkeit (Gülich 1997) oder - mit Feilke (1994: 366) - idiomatische Prägung entsteht zu einem wesentlichen Teil durch den Gebrauch von formelhaften Wendungen unterschiedlichster Typen. Linguistische und kulturhistorische 
Analysen in der HiFoS-Nachwuchsforschergruppe lassen eine ähnliche Relevanz der Formelhaftigkeit für historische Formulierungstraditionen annehmen.

Im vorliegenden Aufsatz folgt der allgemeinen Zusammenfassung der wichtigsten Erkenntnisse im Bereich der gegenwartssprachlich bezogenen Routineformelforschung (Abschnitt 1) ein historischer Umriss (Abschnitt 2). Abschnitte 3 und 4 führen in die Methoden und Techniken der Wissensvermittlung im Frühen Mittelalter ein und bilden eine wichtige Verständnisgrundlage für die Darstellung der Funktionen von historischen Routineformeln in diesem Prozess (Abschnitt 5). Die Analyseergebnisse sind in Abschnitt 6 präsentiert.

\section{Routineformeln in der gegenwartssprachlich bezogenen Phraseologieforschung}

Routineformeln oder anders ausgedrückt kommunikative bzw. pragmatische Phraseologismen gehören seit Beginn der germanistischen Phraseologieforschung in den 60er Jahren des 20. Jahrhunderts zu ihrem Kernbestand. Darunter werden mehr oder weniger feste desemantisierte Wendungen verstanden, mit deren Hilfe immer wiederkehrende kommunikative Handlungen, die so genannten kommunikativen Routinen, bewältigt werden (Burger 2007: 57). Sie können dementsprechend als Entlastung oder Erleichterung bei Formulierungsschwierigkeiten eingesetzt werden.

Für die meisten in der Phraseologieforschung berücksichtigten Standardsprachen können die Routineformeln in vieler Hinsicht als gut erforscht gelten. ${ }^{4}$ Besonders in solchen Kommunikationsbereichen wie informelle und formelle Kontakteröffnungen und -beendigungen wurde die konstitutive Rolle der Routineformeln bereits in mehreren Untersuchungen hervorgehoben: Für direkte und indirekte mündliche Gespräche sowie etwa schriftliche Brieftexte können in ihrer Grundstruktur mehr oder weniger stabile Schemata (formelhafte Texte) festgestellt werden, die mit Hilfe der Routineformeln organisiert sind, einzelne Kommunikationsschritte in diesen Situationen prägen und auf bestimmte soziale Ordnungen verweisen. Die Stabilität geht dabei mit Flexibilität einher: Den Kommunikationsbedingungen entsprechend kann die Struktur und folglich die Verwendung von Routineformeln eine unterschiedliche Entfaltung finden. Sowohl durch Flexibilität, aber auch gleichzeitig durch eine gewisse Stabilität können allgemeine kulturelle Zuweisungen erfolgen, z.B. zu bestimmten historischen Epochen, Institutionen, Altersgruppen, Bevölkerungsschichten, Sprachgemeinschaften usw. (Lüger 2007: 446). Der letzte Aspekt - die kulturelle Geprägtheit der Routineformeln und der interlinguale Vergleich ihrer Verwendung - hat oft den Gegenstand unterschiedlich umfangreicher kontrastiver Untersuchungen 
gebildet (Gülich und Henke 1979/1980; Coulmas 1981; Álvarez de la Granja 2008). Die Wichtigkeit der korrekten Verwendung von Routineformeln für die adäquate soziale Kompetenz in einer Fremdsprache wurde mehrmals seitens der didaktisch ausgerichteten Phraseologieforschung betont (Gülich und Henke 1979/1980; Kühn 1984; Lorenz-Bourjot und Lüger 2001: 85).

Die Forschung unterscheidet grundsätzlich zwischen situationsgebundenen und situationsunabhängigen Routineformeln (Burger et al. 1982: 105; Stein 1995). Für den Gebrauch dieser beiden Typen der Routineformeln als Handlungsmuster ist nicht so sehr das Wissen über ihre semantisch beschreibbare Bedeutung ausschlaggebend, sondern eher das Wissen über die pragmatischen Funktionen, die Routineformeln in der jeweiligen Kommunikationssituation übernehmen. Diese sind in der Regel sehr vielfältig und können bei ein und derselben Formel je nach der Situation und/oder Position im Text variieren. Aufgrund dieser Polyfunktionalität ist in Bezug auf eine Formel eher von einer dominanten Funktion auszugehen, die eng mit einem Spektrum an weiteren Funktionen verbunden ist.

Trotz dieser Schwierigkeit und mit Blick darauf wurden in der traditionellen Phraseologieforschung mehrere Versuche unternommen, Routineformeln nach ihren Funktionen zu klassifizieren. Eine dieser Klassifikationen (Coulmas 1981: 94) sei an dieser Stelle stellvertretend erwähnt. Obwohl sie in der rezenteren Forschung unterschiedlich und kontrovers diskutiert wurde, bildet sie die funktionale Vielfalt der Routineformeln umfassend ab, hat nicht das Ziel,

Tabelle 1. Funktionen der Routineformeln nach Coulmas (1981: 94)

\begin{tabular}{lcl}
\hline soziale Funktionen & diskursive Funktionen \\
\hline- Kontaktfunktion & - & Gesprächssteuerung \\
(Aufmerksamkeitserlangung und & (Eröffnungs- und Einleitungsformeln, \\
-steuerung, Höflichkeitssignale) & Rederechtsverteidigungsformeln, \\
- Stärkung der Verhaltenssicherheit & Signale der Beitragsübergabe, \\
(Zustimmungsformeln, Vorstellungs- & Abschlussformeln) \\
und Entschuldigungsformeln) & - & Evaluation \\
- Schibboleth-Funktion & (psychoostensive Formeln, \\
(gruppenspezifische Anredeformen, & emotive und kognitive Einstellungs- \\
Ausdrücke sozialer Identität) & kundgaben) \\
- Konventionalitätsfunktion & - & Metakommunikation \\
(z.B. Glückwünsche) & (Verständnissicherung, Kommentar- \\
& und Korrekturformeln) \\
& - & Entlastungsfunktion \\
& (Verzögerungsformeln als Anhang- \\
& fragen, Pausenfüller, Hörersignale) \\
\hline
\end{tabular}


sie nach dem Prinzip der Monofunktionalität zu ordnen und eignet sich auch für historische Untersuchungen.

\section{Routineformeln in der historisch ausgerichteten Phraseologieforschung}

Wesentlich weniger Beachtung fanden bis jetzt historische Routineformeln. Für das 19. Jahrhundert hat Linke eine größere Studie (Linke 1996) vorgelegt und die Rolle der Phraseologie, darunter auch Routineformeln, in der bürgerlichen Konversationskultur herausgearbeitet: Im Kontext von Anstandsbesuchen, Tischgesprächen, gesellschaftlichen Anlässen wie Bällen, gemeinsamen Restaurantbesuchen usw. bildeten sich im 19. Jahrhundert phraseologische Wendungen heraus, die sich durch starke soziosemiotische Kraft auszeichnen (Burger 1998: 89-90). Ihre systematische Analyse anhand verschiedener (literarischer und nicht literarischer) Textsorten steht bis jetzt aus. Mit Blick auf das Bestreben, Deutsch rein zu reden und zu schreiben, sind den Routineformeln in den barocken Grammatiken, Kanzleibüchern, Stil- und Schreiblehren einzelne Kapitel gewidmet (Filatkina, im Druck).

Für das Frühmittelalter betont Burger (1977: 17-18) das häufige Vorkommen der Beteuerungsformeln, Ausrufeformeln, Grußformeln und Flüche mit starker Affinität zur gesprochenen Sprache. Eine umfangreiche Sammlung der Beteuerungsformeln primär aus einem Textdenkmal, Otfrids Evangelienbuch, legte Büge (1908) vor. Die geläufige Verwendung solcher Formeln wie das sage ich dir für wahr, das glaubt mir, das mögt ihr gewiss wissen führt Büge (1908: 2) der typischen Einstellung zur formelhaften Sprache am Anfang des 20. Jahrhunderts entsprechend auf volkstümliche Traditionen zurück und sieht darin vor allem „ein Kunstmittel zur lebhaften und kunstvollen Darstellung“ (Büge 1908: 41). Die Routineformeln verteilen sich quantitativ unterschiedlich innerhalb einzelner Kapitel; ihr Einsatz überwiegend bei den Verben des Sagens und Wissens ist vor allem dadurch zu erklären, dass „,der Dichter keinen bequemen Ausdruck wie affirmare, confirmare, arquere, oder wie im Neuhochdeutschen ,versichern', ,behaupten', ,bestätigen“" (Büge 1908: 46) parat hatte und solche Verben umschreiben musste. In den mittelhochdeutschen Texten nehmen Beteuerungsformeln insbesondere in der Funktion der Wahrheitsbeteuerung stark $\mathrm{zu}$.

An den Rändern eines lateinischen Manuskripts der Bibliothèque National in Paris und eines Manuskripts der Vatikanischen Bibliothek sind die so genannten Altdeutschen oder Pariser Gespräche überliefert (Sonderegger 1978: 284-285). Burger hat bereits (1983: 24-34) die Ergiebigkeit dieser Quellen 
für phraseologische Fragestellungen betont, allerdings unterlagen sie bis jetzt kaum einer systematischen Untersuchung. Dieser althochdeutsche (fränkische) Text mit lateinischer Übersetzung wurde von einem Franzosen aus dem Ende des 10. oder Anfang des 11. Jahrhunderts wohl für französische Reisende im deutschsprachigen Gebiet verfasst und besteht aus über 100 Sätzen, Dialogformen und Einzelwörtern. Der Text steht in der Überlieferungstradition der bereits im 3. Jahrhundert bekannten Hermeneumata - Gesprächsbücher der griechischen Konversation für lateinkundige Schüler (Haubrichs und Pfister 1990).

Die in den Altdeutschen Gesprächen überlieferten Sätze vermitteln sprachliches Wissen über die Alltagskommunikation in einer fremden Sprache: Sie stellen meistens imperativische Bitten oder Befehle (1), einige der ersten im Althochdeutschen überlieferten Beschimpfungen (2) sowie fränkische Interjektionen dar, zu denen interessanterweise meistens lateinische Übersetzungen X fehlen (3):

(1) elpe . adiuua. Gimen min ros. i(d est). da $\mid$ mihi $^{m}$. quum. Gimer min scelt . i(d est) scu | Gimer min spera. | Gimer min stap. i(d est) $\mid$ fustum ,Hilfe! Gib mir mein Roß. Gib mir mein Schild. Gib mir meinen Speer. Gib mir meinen Stab.

(2) Vndes ars in tine naso . i(d est) canis culum in tuo naso ,Hundearsch in deine Nase“

(3) Terue geu! Terue nain i. non ,Wahrlich ja! Wahrlich nein

Ähnliches gilt ferner für die sog. Kasseler Glossen im Codex Casselanus theol. $4^{0} 24$, der im 1. Viertel des 9. Jahrhunderts wohl in Regensburg niedergeschrieben wurde (Schröder 1981: 61-63). Sie weisen einen den Altdeutschen Gesprächen ähnlichen Aufbau auf: Auch hier finden sich neben den sachlich angeordneten Einzelwörtern Befehlswendungen und dialogische Routineformeln. Hier werden die mit dem Latein vertrauten (vermutlich italienischen?) Leser in bairischen Routineformeln und Wortformen unterwiesen, so dass sie sich mit Sprechern des Bairischen verständigen können.

(4) Tundi meo capilli skirminfahs ,schere mein Haar Radi me meo colli skirminanhals , schere meinen Hals ${ }^{c}$ Radi meo parba skirminanpart , schere meinen Bart ${ }^{\circ}$ Sic potestfieri somacuuesan ,vielleicht ${ }^{*}$ 
Aus dieser Perspektive des mittelalterlichen Fremdsprachenunterrichts allgemein und des mittelalterlichen DaF-Unterrichts ganz konkret ist das Vorkommen von Beschimpfungen in den Altdeutschen Gesprächen und eine Stelle in den Kasseler Glossen zu verstehen:

\section{(5) Stulti sunt Romani Tole sint uualha ,Dumm sind die Romanen“ Sapienti sunt Paioari spahe sint peigira ,Klug sind die Baiern“}

Penzl (1984: 392) macht darauf aufmerksam, dass es sich aus der Tradition der Hermeneumata ergibt, dass der Lehrer im Unterricht den Schüler über Persönliches ausfragte, ihn beschimpfte und lächerlich machte, um die Schüler zu größerem Fleiß und größerer Aufmerksamkeit zu ermuntern und von Ungezogenheit und Ungehorsam abzuhalten. Unter diesem Gesichtspunkt wären - so Penzl - auch die Routineformeln (5) zu beurteilen: Zur Tradition des Unterrichts gehört, dass der Lehrer seine Überlegenheit in Wissen und die Unwissenheit seines Schülers betont.

Aus heutiger Perspektive interessant ist nicht nur die sprachliche Eigenart des Formulierens im Althochdeutschen, die die Kasseler Glossen sowie die Altdeutschen Gespräche tradieren, sondern auch die Unterschiede der Gesprächskonventionen. Sie kommen am deutlichsten in der Struktur der Gespräche zum Ausdruck: Es finden sich viele Fragen an die Reisenden, die sehr eingehend und detailreich sind. Die Antworten sind dabei eher kurz, sie bestehen oft aus einem Wort oder fehlen ganz. Daraus ist möglicherweise zu schließen, dass es in althochdeutschen Zeiten wohl höflich war, an einen Fremden ganz persönliche Fragen zu richten. Der Fremde aber braucht durchaus nicht genaue Auskunft über seine Reise zu geben, wenn er das nicht wünscht.

Eine dezidierte Verwendung von Routineformeln ist ferner für mittelalterliche Segenssprüche konstitutiv. Trotz der Tatsache, dass Segenssprüche in der letzten Zeit immer öfter als Beispiele der historischen Fachsprache in den Fokus literaturwissenschaftlicher Untersuchungen geraten (Schulz 2000, 2003; Riecke 2004: 92-115), wurden sie bis jetzt kaum textlinguistisch und sprachhistorisch mit Blick auf ihre formelhafte Geprägtheit erforscht. An unterschiedlichen Stellen innerhalb eines Segensspruches kommen unterschiedliche Typen von formelhaften Wendungen, darunter auch Routineformeln, vor, und zwar in den (einleitenden) Historiolae, in den narrativen Passagen sowie in den handlungsorientierten Teilen. Sie fungieren als ein besonderes Mittel der Kommunikation mit dem Leser, als Mittel der Wahrheitsbeteuerung und Vermittlung des mittelalterlichen Wissens. Neben dieser Konstanz bei der Gestaltung der Textsorte an sich weisen Routineformeln in Segenssprüchen im Laufe der Geschichte Variationen auf, die sich sowohl auf der Mikroebene (hier verstanden als die 
Morphosyntax und lexikalische Besetzung der phraseologischen Ausdrucksmittel), als auch auf der Makroebene der Texte (das heißt, ihre innere Organisation) feststellen lassen. Sie verändern sich in ihrer gestalterischen Rolle abhängig von den Veränderungen der Funktion der Textsorte „Segensspruch“ insgesamt. Sie variieren ferner innerhalb der unterschiedlichen Typen der Segenssprüche. ${ }^{5}$

Einen besonderen Status der so genannten pragmatischen Kommentierungen in den mittelhochdeutschen Artusromanen hebt Eikelmann (1999: 304, 2002: 96) hervor. Dabei geht er auf formelhafte Wendungen wie das ist ein altes sprichwort, das ist ein altgesprochenes wort, so sagt man ein, die typologisch als Routineformeln zu klassifizieren sind. Sie signalisieren meistens die Gebräuchlichkeit von Sprichwörtern und tradieren das sprachlich-kulturelle Wissen, das ihre Verwendung voraussetzt. Eikelmann (2002: 96-97) stellt in ihrer Verwendung einen deutlichen Unterschied zur Gegenwart fest: Werden sie heute meist relativierend eingesetzt, hat sie das Mittelalter gezielt und vielgestaltig als Einleitungsformeln, Kommentare und Textparaphrasen genutzt, um Status und Funktion des Sprichworts zu betonen. Auf diese Weise vermitteln die Routineformeln das Wissen über Gebräuchlichkeit, Verbreitung und Wahrheitswert von Sprichwörtern und gewähren Einblicke in ihre kulturellen Verwendungstraditionen.

Friedrich (2006: 33-34, 2007: 1096-1097) zufolge sind für die mittelhochdeutschen. literarischen Texte ferner Bekräftigungs- und Beteuerungsformeln typisch, die Bezug auf Gott bzw. Personen oder Dinge nehmen, die dem Sprecher wichtig sind und auch allgemein als edel, wertvoll oder heilig gelten. Im deutlichen Unterschied zur heutigen Kommunikationspraxis steht die Verwendung von Begrüßungen, die in den mittelhochdeutschen Texten mit Ehrbezeugungen verbunden sind, z.B.: gegruesset siestu, vroewe min, hail sistu, chuninc Marsilie! usw. Einige Routineformeln weisen semantisch-pragmatische Unterschiede auf. So hat die Formel durch got in der Bedeutung ,um Gottes Willen' nicht immer den gegenwärtigen Charakter einer Empörung: Die wörtliche Bedeutung ,für Gott, Gottes eingedenk' verfügt teilweise auch noch über ihre ursprüngliche religiöse Dimension und erinnert den Angesprochenen an dessen Seelenheil, das er mit der rechten Handlungsweise bewahren soll, z.B.: durch got nu senftet iwern haz (Friedrich 2006: 34). Auch die Routineformel gehabe dich wol ist laut Friedrich (2006: 35-36) mittelhochdeutsch vorwiegend Trostformel ,sei guten Mutes; keine Sorge; keine Angst‘. Ihre häufige Verwendung beim Abschied sorgt dafür, dass sie auch (und auf dem Weg zum Neuhochdeutschen fast ausschließlich) als allgemein-wohlwollende Abschiedsformel verwendet wird.

Kaum erforscht bleibt die Rolle der Routineformeln in den historischen Prozessen der Strukturierung und Vermittlung des gelehrten Wissens. Sie soll deshalb im Mittelpunkt des vorliegenden Aufsatzes stehen. 


\section{Wissensvermittlung im Frühmittelalter}

Als Orte der Gelehrsamkeit und der frühmittelalterlichen Wissenschaft sind in erster Linie die Klöster zu nennen. ${ }^{6}$ Im Zentrum der wissenschaftlichen Auseinandersetzung stand die christliche Theologie. Schwerpunkt der klösterlichen Schulausbildung stellt daher die Vermittlung biblischer und theologischer Inhalte dar, allem voran die Auslegung biblischer Texte. Seit der Admonitio Generalis, die Karl der Große 789 erlassen hatte, um die professionelle Ausbildung des Klerus zu verbessern, ${ }^{7}$ standen sowohl die Bibel selbst als auch Schriften der Kirchenväter sowie (spät)antiker Philosophen wie Boethius, Martianus Capella oder Terenz auf den Lehrplänen der Klosterschulen. Die antike Tradition der Sieben Freien Künste bildete seit der Admonitio Generalis wieder die Basis des lateinischsprachigen Unterrichts. In den Fächern des Triviums (Grammatik, Rhetorik und Dialektik) wurden die lateinischen Sprachkenntnisse vertieft und das Textverständnis gefördert. Im darauf aufbauenden Quadrivium, bestehend aus den naturwissenschaftlichen Fächern Arithmetik, Musik, Astronomie und Geometrie, wurden die Schüler auf das Studium der Theologie vorbereitet (Ochsenbein 1991: 137).

Allmählich wurde die Bedeutung des Trivium-Studiums weiter ausgebaut, diente es doch vornehmlich dem Verständnis und der christlichen Exegese der Bibel. Um einen festen Grundstock an Allgemeinwissen aufzubauen, beschäftigten sich die Schüler in den Trivium-Fächern in erster Linie mit christlichen philosophischen und theologischen Texten, die im Laufe der Zeit auch um Schriften antiker, nicht christlicher Autoren wie Ovid, Vergil oder Aristoteles erweitert wurden. ${ }^{8}$

Die schulische Wissensvermittlung erfolgte Baldzuhn (2002) zufolge bis ins Spätmittelalter mündlich. Schriftlichkeit und damit das stille Lesen statt des Hörens eines Lehrvortrags drang erst spät in den Unterricht ein. Die überlieferten Texte, die für den Schulkontext angefertigt wurden, stellen also Zeugnisse eines spezifischen Verhältnisses zwischen Mündlichkeit und Schriftlichkeit dar. Baldzuhn (2002: 167) bemerkt hierzu: „Gerade die gegen das Licht der mündlichen Lehrtradition betrachteten schriftlichen Quellen werden darin als Vermittlungsprodukt sichtbar, in dem oral und literal geprägte Kommunikationsstrategien ineinander greifen."

Neben der Wissensvermittlung im schulischen Kontext steht die Auseinandersetzung mit theologischen oder säkularen Themen wie beispielsweise der Bibelexegese, der theologischen Dogmatik, der christlichen Ethik, der Hagiographie oder der Historiographie, die in ihrer Intention an ein gebildetes klerikales Fachpublikum gerichtet waren. ${ }^{9}$ Wissenschaftliche Werke des Frühmittelalters entstanden primär durch Kommentierung und/oder Kompilation, und stellten 
sich bewusst in den Dienst der christlichen Aneignung des Bildungsgutes (Goetz 2003: 256). Bildung und Wissenschaft hatten im Frühmittelalter also keinen Selbstzweck, sondern waren immer Instrumente der Vermittlung christlicher Inhalte. Es entstand dennoch - oder gerade deshalb - in allen Wissensgebieten eine reichhaltige lateinische Literatur, in der zwar vieles aus bereits vorhandenen Inhalten neu zusammengestellt und der Zeit angepasst wurde, gleichzeitig aber auch zahlreiche eigenständige Werke entstanden, die von der Bildungsgesellschaft des Frühmittelalters aufgenommen und in den wissenschaftlichen Diskurs überführt wurden.

Einen weiteren Bereich der Wissensvermittlung stellte die Unterweisung der Bevölkerung in Fragen des christlichen Glaubens dar: Das Ziel dieser Bestrebungen, die in erster Linie vom niederen Klerus getragen wurden, war es, essentielle Glaubensinhalte und Kenntnisse zumindest des Vaterunsers und des Glaubensbekenntnisses weiterzugeben (Haubrichs 1995: 58). Dabei richteten sich diese Bemühungen nicht nur an eine nicht-christliche Bevölkerung, wie sie bis ins 9./10. Jahrhundert noch in Teilen des Frankenreichs zu finden war (Kahl 2000: 669), sondern auch an eine bereits nominell dem Christentum angehörende Zielgruppe.

\section{Wissensvermittelnde volkssprachige Texte im Frühmittelalter}

Wie bereits erwähnt, fand die schulische Unterweisung des monastischen Klerus in den Klosterschulen statt. Für die Ausbildung nach dem Bildungsprogramm der Sieben Freien Künste wurde ein Kanon an grundlegenden Texten herangezogen, die einerseits auf spätantike und/oder frühchristliche Autoren wie Boethius, Martianus Capella, Cassiodor oder Prudentius (Glauche 1970: 76; Ochensbein 1991: 138) zurückgingen und andererseits aus der Bibel stammten wie beispielsweise der Psalter, der eine zentrale Stellung nicht nur im monastischen Schulunterricht einnahm (Ochsenbein 1991: 140).

Diese Texte wurden in der Unterrichtssprache Latein gelesen und behandelt. Eines der Ziele, die die Ausbildung der jungen Mönche hatte, war die Vermittlung des Lateinischen als Sprache der Bibel und somit als Wissenschaftssprache schlechthin. Erst im beginnenden 11. Jahrhundert wurde ein Teil dieser Werke erstmals durch den St. Galler Schulmeister Notker den Deutschen in die Volkssprache übersetzt, um im Unterricht Verwendung zu finden. Doch Notker beließ es nicht bei einer reinen Übersetzung, sondern versuchte die komplexen Inhalte auch in der Volkssprache zu erklären. In einem Brief an den Bischof Heinrich von Sitten rechtfertigte Notker sein Vorgehen: 
Scio tamen, quia primum abhorrebitis quasi ab insuetis, sed paulatim forte incipient se commendare vobis, et praevalebitis ad legendum et ad dinoscendum, quam cito capiuntur per patriam linguam, quae aut vix aut non integre capienda forent in lingua non propria.

,Ich weiß dennoch, dass Ihr vielleicht zuerst erschrecken werdet, wie vor etwas Ungewohntem, aber allmählich werden sie vielleicht anfangen, sich euch zu empfehlen, und Ihr werdet beim Lesen und beim Erkennen durchdringen, wie schnell sie verstanden werden durch die Vatersprache, welche sie entweder mit Mühe oder nicht anständig beim Erfassen durchdringen können in der nicht eigenen Sprache.‘

(Zitat und Übersetzung nach Hellgardt 1979: 13)

Notkers Intention war es also, den Zugang zu intellektuell anspruchsvollen Inhalten über die Muttersprache zu erleichtern. Das „Unerhörte“ seines Vorgehens war es dabei, dass er nicht nur eine Übersetzung oder Glossierung anbot, sondern auch die Erklärung in der Volkssprache bereitstellte. ${ }^{10}$ Notker übersetzte und kommentierte während seiner Zeit als Schulmeister unter anderem Texte, die im Dialektikunterricht herangezogen wurden, wie die aristotelischen Schriften Categoriae, De interpretatione, die in den Übersetzungen des Boethius vorlagen und weitere Schriften zur Logik wie De syllogismis, De partibus logicae und De definitione. Hinzu kommt die Übersetzung und Kommentierung eines Teils des allegorischen Werkes De nuptiis Philologiae et Mercurii des Martianus Capella. Diese Schriften wurden in Notkers Unterricht eingesetzt, worauf auch die entsprechende Aufbereitung der Texte hinweist: Einerseits wurde der lateinische Text so bearbeitet, dass die Schüler leichter einen Zugang zur syntaktischen Struktur fanden, andererseits wurde der Text mithilfe von formelhaften Wendungen, mithin Routineformeln, aber auch durch Sprichwörter angereichert, damit Wissensinhalte besser vermittelt werden konnten (Filatkina u.a. 2009).

Daneben wurden in den Klöstern auch Werke verfasst, die sich der Bibelexegese widmeten. Trotz der Dominanz des Lateinischen auf dem Gebiet der Bibelexegese entstanden seit der karolingischen Renaissance auch volkssprachige Texte, die nicht allein der Missionierung der Bevölkerung dienten, sondern auch für die Rezeption innerhalb des Klosters intendiert waren. So nimmt man an, dass beispielsweise das Evangelienbuch Otfrids von Weißenburg, das um 860/70 entstand, im Kloster sowohl der Privatlektüre der Mönche als auch der Tischlesung im Refektorium diente (Green 1987: 769). Die einzelnen Abschnitte dieses das Leben Jesu nacherzählenden Werkes werden immer wieder durch exegetische Exkurse unterbrochen, in denen die narrativen Inhalte erklärt und ausgelegt werden. Diese Exkurse dienen also der Vermittlung theologischer Lehren, durch die das Heilswirken Jesu deutlich gemacht wird. Es entsteht eine enge Verklammerung 
von Wiedergabe und Deutung des biblischen Textes, wie Hellgardt (1981: 146) feststellt.

Während sich mit dem Ende der karolingischen Herrschaft im 10. Jahrhundert eine Rückkehr zur Bildungssprache Latein abzeichnete, ${ }^{11}$ setzte um 1000 mit der kommentierenden Übersetzung des Psalters durch Notker den Deutschen allmählich wieder eine volkssprachliche Schriftlichkeit ein, die freilich noch immer in engem Verhältnis zum Lateinischen stand. Mit der auf Augustinus, Cassiodor und Hieronymus fußenden Auslegung des Psalters als einem Grundtext der theologischen Bildung eines Klosterschülers versuchte Notker die vielschichtigen Inhalte der Bibelexegese für den schulischen Gebrauch aufzubereiten.

Die um 1060/65 entstandene Auslegung des Hohenliedes des Ebersberger Abtes Williram stellt eine weitere Form der exegetischen Bearbeitung eines biblischen Textes in der Volkssprache dar. Ähnlich wie Notker verwendete Williram eine deutsch-lateinische Mischsprache, die wichtige Inhalte zwar auf Deutsch darbietet, Fachtermini jedoch in der lateinischen lingua sacra belässt. Auch er stützte sich in seiner Auslegung des Hoheliedtextes auf anerkannte Kirchenlehrer wie Haimo von Auxerre und übertrug ihre Gedanken gekonnt in die Volkssprache. Der Rezipientenkreis der Hoheliedparaphrase ist jedoch - anders als bei Notkernicht im schulischen Umfeld zu suchen, sondern vielmehr bei den lateinisch gebildeten Gelehrten der Zeit (Zerfaß 1995: 204). So stellte Williram neben seine deutsch-lateinische Prosaparaphrase eine kunstvolle lateinische Versparaphrase, die der intendierte Leser in Zusammenspiel mit dem Prosatext rezipieren sollte (Zerfaß 1995: 16). Gleichwohl sollte auch hier Wissen vermittelt werden: Wissen um die Auslegungspraxis des als rätselhaft empfundenen Hoheliedes, womit Williram einem Bedürfnis seiner Zeit nachkommt (Grabmeyer 1976: 397).

Interessant erscheint aus phraseologischer Sicht, dass die Wissensinhalte bei diesen doch sehr unterschiedlichen Texttypen vielfältig mit formelhaften Wendungen vermittelt werden. Im Folgenden soll anhand exemplarisch ausgewählter Texte, nämlich den Schultexten Notkers, den Exegesepassagen im Evangelienbuch Otfrids und der Hoheliedparaphrase Willirams untersucht werden, wie Routineformeln zur Textgliederung und Wissensvermittlung eingesetzt werden.

\section{Strategien der Wissensstrukturierung und -vermittlung in althochdeutschen Texten unter Einsatz der Routineformeln}

Die zum Teil hochkomplexen Inhalte der exegetischen oder schulischen Literatur, die in die Volkssprache transferiert wurden, mussten von den Bearbeitern so aufbereitet werden, dass sie von ihren intendierten Rezipienten verstanden 
werden konnten. Der Textautor bzw. -bearbeiter tritt also als Vermittler zwischen den Textinhalten und den Rezipienten. Auffällig hierbei ist die ausgiebige Verwendung von Routineformeln, die gerade diesem Zweck der Aufbereitung, der Textgliederung und der Wissensvermittlung dienen. Die hier vermittelten Inhalte stellen im Gegensatz zu den bereits besprochenen Beispielen kein Alltags-, sondern vielmehr eine Form von Spezialwissen dar, das an einen ganz speziellen Kreis von Empfängern, nämlich in der Regel an Klosterschüler und/ oder bereits gebildete Kleriker gerichtet ist.

Problematisch erweist sich die Frage nach der Rezeptionssituation der jeweiligen Texte, die einen Einfluss auf die Beurteilung der pragmatischen Dimension der eingesetzten Routineformeln hat: War der Text in erster Linie zum mündlichen Vortrag gedacht wie etwa für die Verwendung im Unterricht, wodurch die schriftlich belegten Routineformeln Sprechakte innerhalb einer medialen Mündlichkeit darstellten (vgl. Baldzuhn 2002: 164)? Oder war der Text zur stillen Lektüre intendiert, ohne die Möglichkeit eines mündlichen Vortrags zu berücksichtigen? Sichere Aussagen lassen sich hierzu nicht machen, beide Möglichkeiten müssen aber für jeden Text berücksichtigt werden, wie Green in Bezug auf das Evangelienbuch Otfrids feststellt (Green 1987: 769).

Deshalb erscheint ein textbezogener Ansatz, der die Routineformeln innerhalb ihres situativen Kontextes interpretiert, notwendig. Im Folgenden wird anhand ausgewählter Stellen bei Otfrid, Notker und Williram demonstriert, wie sie in ihren Texten Routineformeln einsetzen, um ihre Inhalte zu strukturieren und für den Rezipienten aufzubereiten. Wie oben bereits erwähnt, ist die Bestimmung einer dominanten pragmatischen Funktion in einigen Fällen nicht eindeutig, da Routineformeln polyfunktional sein können. Eine Beschreibung der Formeln erfordert daher immer auch die Einbeziehung ihres aus dem Kontext ableitbaren Funktionsspektrums (Filatkina 2007: 140). Im Sinne der in Abschnitt 1 zusammengefassten Überlegungen wird bei der folgenden Darstellung der verwendeten wissensstrukturierenden Routineformeln in Anlehnung an die Klassifikation Coulmas' eine dominante pragmatische Funktion angegeben, die jedoch erweitert wird durch ein Spektrum an zusätzlichen Funktionen, die die Formeln innerhalb des jeweiligen Kontextes zusätzlich erfüllen. ${ }^{12}$

\subsection{Aufmerksamkeitssteuernde Routineformeln}

Im Anschluss an die Erzählung von der Hochzeit in Kanaa, bei der Jesus Wasser zu Wein verwandelte, deutet Otfrid dieses Geschehen in einem SpiritualiterKapitel aus: Er beginnt seine Auslegung mit der Routineformel fernemet sar in rihti, mit der er einerseits die Aufmerksamkeit seiner Rezipienten für sich 
beansprucht, andererseits aber die erste wichtige Information einleitet, nämlich dass im theologischen Sinn Christus selbst für den Bräutigam steht:

(6) Fernem(et) sar in rihti . thar (Christus) ther brutigomo si $\mid$ ioh druta sine in lante . zi theru bruti ginante

,Vernehmt sodann direkt, dort ist Christus der Bräutigam und seine Vertrauten im Land. zur Braut bestimmt.

(Otfrid, Evangelienbuch 53v, II 9, 7; ID 9691)

Gerade weil diese Routineformel bei der Vermittlung der für das mittelalterliche Wissensbild wichtigen Kenntnisse und Überzeugen eingesetzt wird, wird sie oft mit den Wahrheitsbeteuerungen in alauuari oder giuuisso kombiniert:

(7) Firnim in alauuari . thaz got ther fater uuari $\mid$ ioh thaz kind eino . kristan bezeino

,Vernimm wahrheitsgemäß, dass Gott der Vater ist und dass das einzige Kind . Christus bezeichnet."

(Otfrid, Evangelienbuch 55r, II 9, 75; ID 9697)

Auch Notker verwendet diese Formel mit Vorliebe in seinen Schultexten. In der Bearbeitung des auf Aristoteles zurückgehenden Werkes Categoriae erklärt Notker die Unterscheidung der ontologisch selbstständigen und der sekundären Substanzen:

(8) Et cu(m) aliqua(m) ar|bore(m) reddideris. man ifestius assignabis cu(m) ar|bore $(m)$ assignabis qua(m) arbustu(m). Zéigôst tû uuáz | éin réba sî. táz tuôst tû báz póum chédendo. | tánne dáz in érdo stât. An érdo stât oúh chrûit $\mid$ unde speid. Amplius. Ferním nóh mêr. Prime | substantie $p$ (ro)pterea $q(u o) d$ aliis om(n)ib(us) subiacent. (et) $\mid$ alia om(n)ia dehis om(n)ibus p(re)dicent(ur). aut in eis s(unt). idcir|co maxime substantie $d($ icu)n(tur) .

,Und wenn du irgendeinen Baum wiedergibst, wirst du ihn deutlicher bezeichnen, wenn du ,Baum' sagst, als wenn du, baumartig 'sagst. Zeigst $\mathrm{du}$, was ein Weinstock sei, dann tust du es besser, indem du ,Baum' sagst, als (indem du sagst), das, (was) auf der Erde steht'. Auf der Erde steht auch Kraut und Busch. Ferner: Vernimm noch mehr: Die prime substantie (die ersten Substanzen) werden so genannt, weil sie allen Dingen zu Grunde liegen und alles andere von ihnen ausgesagt wird oder in ihnen ist. Deshalb werden sie maxime substantie (oberste Substanzen) genannt.

(Notker, Categoriae (Hs. B, CSg 818) 23, 1; ID 5140) 
Auch hier wird die Formel fernim nóh mêr eingesetzt, um den Rezipienten anzusprechen, seine Aufmerksamkeit weiter in Anspruch zu nehmen, aber darüber hinaus, um eine vertiefende Information einzuführen, die sich direkt auf das Vorausgehende bezieht und dieses ergänzt. Damit wird der Rezipient aufgefordert, sich diese Informationen zu merken.

Zudem kann diese Formel auch eine belehrende Funktion aufweisen, wenn sie etwa als Einleitung einer Zusammenfassung der vorausgehenden Aussagen verwendet wird und hier noch einmal ganz dezidiert auf die wichtigen Lehrinhalte verweist, wie etwa in folgendem Beispiel:

(9) keméinlîcho uernín uóne állen daz óuh fóre ge|ságet ist. daz tu e(ss)e unde non esse háben sólt fúre $\mid$ subiecta. unde affirmatione $(m)$ ih méino est. ioh ne|gatione $(m)$ ih méino no(n) est. sólt tu légen zû demo mo|do. ,Gemeinhin vernimm von allen, was auch vorher gesagt wurde, dass du esse und non esse für subiecta halten sollst und die affirmatio, ich meine est und die negatio, ich meine non est, sollst du zu dem modus hinzusetzen.

(Notker, De interpretatione 219, 12; ID 13086)

Die Formel firnemet/firnim mitsamt ihrer Varianten ist für das Althochdeutsche bislang ${ }^{13} 43 \mathrm{Mal}$ in der HiFoS-Datenbank belegt, wobei sie in all diesen Belegen eine vergleichbare Funktionalität aufweist: Ihren Einsatz findet die Formel in erster Linie in wissensvermittelnden Kontexten, in denen die Aufmerksamkeit des Rezipienten errungen wird bzw. er dazu aufgefordert werden muss, sich wichtiges Wissen zu merken. Vor allem für Notker und Otfrid ist die Verstärkung durch die Wahrheitsbeteuerung kennzeichnend. Williram hingegen kennt diesen Einsatz nicht und benutzt die Routineformel allgemein seltener (auf ihn gehen nur drei Belege zurück). Die Struktur der Wendung ist auf $n u$ uernemet, nv uernim bzw. nu uernemet diu mâre reduziert und bezieht sich in ihrer Funktionalität verstärkt auf Aufmerksamkeitssteuerung und Einleitung. Die zurückhaltendere Verwendung der Formel bei Williram ist möglicherweise durch den Rezipientenkreis, an den sich der Autor wendet, bedingt: Anders als bei Notker richtet sich Williram in erster Linie an ein Fachpublikum, an ausgebildete Theologen, die mit der Exegese des Hoheliedes vertraut sind. Die Aufforderung, sich eine Information im Sinne einer schulischen Lektion zu merken, tritt hier eher in den Hintergrund.

\subsection{Belehrende Routineformeln}

Belehrende Funktion hat die Formel ih sagen dir, die verwendet wird, um einer Aussage Nachdruck zu verleihen, um zu zeigen, dass das Nachfolgende von 
hoher Bedeutung ist und daher vom Rezipienten mit besonderer Umsicht zur Kenntnis genommen werden soll. So erklärt Otfrid in Rekurs auf Joh. 1,8, dass Johannes der Täufer nicht mit dem Messias verwechselt werden dürfe, sondern als dessen Vorbote zu verstehen sei:

(10) Ni uuas er thaz lioht ih sagen thir ein. thaz thar liutin irskein $\mid$ suntar quam sie manoti . ioh thanana in gisag(et)i

,Nicht war er [Johannes] das Licht, ich sage dir nur, welches den Leuten da erschien, sondern er kam, um sie zu mahnen und um ihnen davon zu sagen.

(Otfrid, Evangelienbuch 43r, II 2, 11; ID 9611)

Mit der Formel ih sagen thir ein unterstreicht Otfrid die Aussage und belehrt seinen Rezipienten, indem er sich direkt an ihn wendet. Durch diese direkte Ansprache wird aber wiederum die Aufmerksamkeit des Rezipienten auf das Nachfolgende gesteuert und der Kontakt zwischen Rezipienten und Vermittler erneuert. Somit hat die Formel auch eine Kontakt sichernde Funktion. In diesem Funktionsspektrum tritt die Formel auch in Notkers Bearbeitung von De interpretatione auf:

(11) dico (et)ia(m) no(n) fieri una(m) affirmatione(m) (ue)l ne|gatione(m). si unu(m) nom(en) co(m)mune positu(m) sit mult|is rebus. (et) si ex illis $n(o n)$ sit unu(m). Íh ságo dir daz $\mid$ ouh tánnan $\hat{u} z$ ein affirmatio alde ein nega|tio neuuirdet. ube éin nome(n) geméine fúnde $\mid$ ist mánigen díngen diu éin specie (m) nîeht ke|uuúrchen nemúgen

,Ich behaupte auch, dass es nicht eine affirmatio (Bejahung) oder negatio (Verneinung) geben wird, wenn ein gemeinsamer Name vielen Dingen gegeben wird und aus diesen nicht eines existiert. Ich sage Dir, dass daraus nicht eine affirmatio (Bejahung) oder eine negatio (Verneinung) wird, wenn ein gemeinsames nomen (Name) mehreren Dingen gegeben wird, die nicht eine specie (Gattung) bilden können.'

(Notker, De interpretatione 201, 11; ID 4030)

Notker wendet sich hier direkt an den Rezipienten, womit sich ein Unterschied zur lateinischen Stelle, die er übersetzt, ergibt, die lediglich dico ,ich sage, ich benenne' aufweist, also keine Routineformel mit explizit dialogischer Funktion verwendet. Dadurch verstärkt Notker die belehrende Funktion der Routineformel.

Die Form ih sago dir und ihre Varianten sind insgesamt 82 Mal für die althochdeutsche Überlieferung in der HiFoS-Datenbank belegt. In den narrativen Texten wie dem Evangelienbuch Otfrids ist sie einerseits in der Erzählerrede 
verwendet und dient dem Erzähler in seiner Funktion als Vermittler der direkten Hinwendung an den Leser/Hörer. Andererseits wird sie aber auch in der Figurenrede eingesetzt, in erster Linie, wenn es um die Einleitung einer wichtigen Aussage geht. In den meisten Fällen wird diese Formel verwendet, wenn sich Jesus an seine Jünger wendet und ist somit eine Übernahme der im Neuen Testament sehr häufigen Formel amen dico vobis (vgl. Matth. 6,2; 6,5; 6,16 u.ö.). In ihrer pragmatischen Verwendung ist die Formel weitgehend konstant. Interessanterweise wird sie in anderen hier nicht berücksichtigten Texten (so z.B. im althochdeutschen Tatian) konsequent durch die Routineformel uuâr uuâr quidu ih iu ersetzt. Es bleibt zu untersuchen, ob dies nicht auf einen semantischen Unterschied zwischen den Verben quedan und sagên zumindest im Bereich der formelhaften Sprachen hindeutet.

Otfrid führt in einem Spiritualiter-Kapitel seine Überlegungen zur Nächstenliebe als die höchste Form der Liebe aus:

(12) Nist thiu minna sumirih . kreftin anderen gilih $\mid$ giuuisso unizist thu thaz. thia uuir heizen karitas

,Diese Liebe ist wahrlich nicht anderen Kräften gleich, wisse das gewiss, die wir Caritas nennen.

(Otfrid, Evangelienbuch 168r, V 12, 80; ID 11838)

Mit Hilfe der Routineformel giuuisso uuizist thu thaz belehrt Otfrid seinen Rezipienten, indem er bekräftigend darauf hinweist, dass es sich um eine wichtige Information handle. Durch die direkte Ansprache des Hörers/Lesers sichert Otfrid einerseits den Kontakt zu diesem und betont zusätzlich die Bedeutung der Aussage. Die Formel inklusive ihrer Varianten ist in der HiFoS-Datenbank $63 \mathrm{Mal}$ belegt. Sie kommt hierbei in Kontexten vor, in denen der Rezipient über einen wichtigen Umstand belehrt werden soll. Bei Notker und besonders Otfrid wird diese Routineformel wie das oben gezeigte Beispiel uernemet/uernim mit den Wahrheitsbeteuerungen giuuisso, giuuisso [...] âna uuân, gimuato, in alauuâr, ana zuiual, zi uиâre, in uuâra, giuuâro kombiniert.

Von diesen primär an den Rezipienten gerichteten Verwendungssituationen sind Stellen zu unterscheiden, in denen die Routineformel innerhalb der Figurenrede in den narrativen Passagen in der Form ih uиeiz uuola vorkommt. Sie drückt die Belehrung durch den Rekurs auf den autoritären Status des Autors aus, signalisiert die Übernahme der Sprecherrolle durch ihn und leitet Wissensinhalte ein, denen eine besondere Wichtigkeit zugesprochen wird. Diese Verwendung ist vor allen für Williram kennzeichnet, obwohl er auch hier nach wie vor zurückhaltend ist: Die Formel ist bei ihm $6 \mathrm{Mal}$ in der invarianten oben angeführten Form belegt. 


\subsection{Hinweisende Routineformeln}

Ebenfalls belehrende, aber im Wesentlichen auch hinweisende Funktion haben die Formeln hier ist ze uuizzen und hier ist ze merchene, die sowohl von Notker als auch von Williram eingesetzt werden. Diese Formeln machen auf einen bemerkenswerten Aspekt im Text aufmerksam. So beispielsweise in der Hoheliedparaphrase Willirams, in der ein Vers zweimal im Bibeltext vorkommt, aber je nach Kontext eine andere Interpretation erfährt, worauf Williram mit der Formel hinweist:

(13) DAZ sélba uérs stêt oûh $\mid$ da uora . ad tale signv(m). $\mid \underline{\text { Daz ẹina íst ábo hîe }}$ $\underline{z e} \mid$ mérchene . daz daz êrera | uérs hôret ad primitiua(m) êccl(esi)am | de iudęis collecta $(m)$ !

,Derselbe Vers steht auch davor, bei einem solchen Zeichen. Das eine ist aber hier anzumerken, dass sich der erste Vers auf die erste versammelte Kirche der Juden bezieht. ‘

(Williram, Hoheliedkommentar 21v, 33c; ID 3018)

Diese Formel ist für das Althochdeutsche in der HiFoS-Datenbank zwar nur einmal belegt, wird aber im Frühneuhochdeutschen sehr häufig in wissensvermittelnden Texten wie beispielsweise in der Historiographie eingesetzt. Bislang sind 17 Belege dieser Formel und ihrer Varianten für das Frühneuhochdeutsche in der Datenbank verzeichnet.

In einem sternenkundlichen Exkurs, in Notkers Martianus CapellaBearbeitung, wird das Sternbild des Bärenhüters (Boötes) beschrieben. Um hier wissenswerte Hintergrundinformationen anzufügen, die es dem Rezipienten ermöglichen, das Sternbild einzuordnen, verwendet Notker die Routineformel hier ist ze uuîzenne.

(14) Sî rûor (et) boote (m) án dien |áhselon . hárto skîmbariu éinahálb . únde áber ánderhálb tún|cheliu. Hier íst ze unîzenne . dáz er (ét)elichiu nórd zéichen $\mid$ únde (ét)elichiu súnt zéichen némmendo álliu zéichen ferfâ|h(et). ,Sie [das Sternbild der nördlichen Himmelskrone] berührt Boötes an den Achseln, sehr Leuchtende auf der einen Seite und aber eine Dunkle auf der anderen Seite. Hier ist zu wissen, dass er [Boötes] etliche nördliche Sternbilder und etliche südliche Sternbilder nennend alle Sternbilder umfasst. ‘

(Notker, Martianus Capella 87, 13; ID 3532)

Die Wendung fungiert hier somit als hinweisende Einleitungsformel, mit der der Rezipient über einen weiteren Wissensaspekt belehrt wird. Sie ist insgesamt 
$4 \mathrm{Mal}$ in der HiFoS-Datenbank belegt. Alle Belege stammen aus den Schultexten Notkers und weisen eine ähnliche Funktionalität wie im hier beschriebenen Beleg auf.

\subsection{Erklärende/ausdeutende Routineformeln}

Die exegetischen wie auch die philosophischen Texte weisen immer wieder schwer verständliche Passagen auf, die eine große Herausforderung an die Rezipienten stellen. Die Bearbeiter dieser Texte wie Notker und Williram sind daher bemüht, solche Stellen zu erklären oder durch veranschaulichende Beispiele nachvollziehbar zu machen, unbekannte lateinische Wendungen, Fachwortschatz für den Rezipienten zu übersetzen oder aber zu paraphrasieren. Um solche erklärenden Zusätze einzuleiten, werden wiederum Routineformeln wie daz chît oder ich meino verwendet.

Der sehr bildhafte Text des Hoheliedes muss beispielsweise von Williram in seiner Auslegung immer wieder mit konkreten Bedeutungen verbunden werden, damit der Rezipient die Aussage des Bibeltextes versteht. So auch in folgendem Beispiel, in dem es um einen Vergleich der Haare des Bräutigams mit aufrecht stehenden Palmenwedeln geht. In der folgenden Auslegung lässt Williram den Bräutigam, der mit einer christlichen Auslegungstradition folgend als Christus interpretiert wird, ${ }^{14}$ vom ewigen Heil sprechen, das erst erfüllt werden kann, nachdem Christus die Passion auf sich genommen habe:

(15) Alle dîu gnâda. | dîe íh dír hában gehêiz|zan . dîu neuuírdet ê nîeth | uólliclîcho gelêistet! ê ih $\mid$ gestîgon ûffe dén pálm|bôum . ih mêino daz crûce.

,Alle Gnade, die ich [= Christus] dir habe verheißen, die wird nicht eher völlig geleistet, bevor ich auf den Palmbaum gestiegen bin, ich meine das Kreuz."

(Williram, Hoheliedkommentar 53v, 27c; ID 3079)

Die Routineformel ich meino leitet hier also die Auslegung des metaphorisch verwendeten Begriffs palmbôum ,Palme ' ein und stellt den Bezug zwischen der alttestamentarischen Hoheliedstelle zur Passion Christi her.

Auch Notker verwendet sehr häufig in seinen schuldidaktischen Texten erklärende Formeln, um genauer auszudrücken, was mit einer bestimmten vorausgehenden Wendung gemeint ist. Im folgenden Beispiel aus der Martianus Capella-Bearbeitung wird die Göttin Diana - für das christlich geprägte Frühmittelalter keine geläufige mythologische Person - eingeführt und ihr Name mit Hilfe einer im Mittelalter beliebten Form der etymologischen Herleitung erklärt: 
(16) Áber luna héizet diana . sámo so du|ana . uuánda sî duob(us) te(m)porib (us). íh méino dieb(us) ac noctib(us) | zeóugon ist .

,Aber Luna heißt Diana, gleichsam Duana, weil sie duobus temporis (zu zwei Zeiten), ich meine tagsüber und nachts, zu sehen ist.

(Notker, Martianus Capella 146, 16; ID 4672)

Mit der Formel ih meino wird hier erklärt, welche zwei Zeiten gemeint sind, um dem Rezipienten das Verständnis dieser etymologischen Herleitung zu erleichtern. Ih meino zählt zu den hochfrequenten Formeln, die zur Wissensvermittlung im Althochdeutschen genutzt werden. Sie weist auch kaum Varianten auf, nur in sehr wenigen Fällen ist ein Akkusativobjekt (ih meino des) hinzugefügt. Sie ist bislang $92 \mathrm{Mal}$ für das Althochdeutsche in der HiFoS-Datenbank belegt.

Eine ähnliche Funktion wie ih meino weist die Formel daz chit auf, die sowohl bei Notker als auch bei Williram zur Einleitung einer Erklärung oder einer Übersetzung verwendet wird. So leitet $d a z$ chît in folgendem Beleg aus der Psalterbearbeitung Notkers eine Auslegung des Bibeltextes ein:

(17) DOMINE DEVS MEVS IN TE | speraui. saluum me fac ex omnibus p(er)sequenti|bus me et libera me. An dih trúhten gedingta ih $\mid$ iêo . halt mih . daz chît . nére mih fóne mînen fienden . unde | lôse mih.

,Herr, mein Gott, auf dich hoffte ich. Mache mich heil von allen Verfolgern und befreie mich. Auf dich, Herr, habe ich stets gehofft, bewahre mich, das heißt: Rette mich von meinen Feinden und erlöse mich.

(Notker, Psalmenauslegung 21,13 (Psalm 7,2); ID 3937)

Durch die Routineformel daz chît wird noch einmal genau erklärt, was mit dem althochdeutschen Wort haltan in der Bedeutung ,bewahren, beschützen“ gemeint ist. Hier wird also ein quasi-synonymer Begriff durch die Formel daz chît eingeführt, der das Verständnis dieser Textstelle erleichtern soll.

In folgendem Beleg aus der Hoheliedparaphrase Willirams leitet die Formel eine Übersetzung aus dem Lateinischen ein, die ihrerseits eine Übersetzung aus dem Hebräischen ist. Es geht um die etymologisierende Herleitung von biblischen Personennamen, in diesem Fall des Namens David:

(18) Dauîdis námo | uuírt gántfrístet . manu for|tis! daz quît . ármstrénger. ,Davids Name wird gedeutet starke Hand, das heißt Starkarmiger.' (Williram, Hoheliedkommentar 27v, 18c; ID 3028)

Auch Notker nutzt diese Formel, um Übersetzungen einzuleiten, beispielsweise in seiner Martianus Capella-Bearbeitung, u.a. wenn er Begriffe, die bei Martinus 
griechisch wiedergegeben sind ins Lateinische übersetzt. In den meisten Fällen unterlässt er jedoch eine weitere Übersetzung ins Deutsche. Umso bemerkenswerter ist die Verwendung der volkssprachigen Routineformel daz chît für den Transfer vom Griechischen ins Lateinische:

(19) Téro sláhto sint $\mid$ óuh egipani . dîe fóne egea gehéizen sint túz chît capra ,Dieser Art sind auch die Egipanen, die nach Egea benannt sind, das heißt capra (Ziege).

(Notker, Martianus Capella 144, 11; ID 4669)

Diese Formel, die ebenso wie ih meino fast ohne Varianz auftritt, ist gegenwärtig insgesamt $122 \mathrm{Mal}$ in der HiFoS-Datenbank belegt. Auffällig ist, dass sie fast ausschließlich von Notker und Williram verwendet wird, also in den erklärenden, deutenden und auslegenden Kontext der Schul- und Exegeseliteratur verweist.

\section{Fazit}

Die hier aufgeführten Routineformeln stellen nur einen Bruchteil der im Althochdeutschen vorhandenen und gebräuchlichen pragmatischen Formeln da, die zur Wissensaufbereitung und -vermittlung dienten.

Es zeigt sich, dass die althochdeutschen Autoren ganz gezielt Routineformeln einsetzten, um Wissensinhalte aufzubereiten und ihren Rezipienten nahezubringen. Dabei dienen die Routineformeln auch in lateinisch-deutschen Textensembles wie den Notkerschen Schultexten oder der Hoheliedparaphrase Willirams dazu, zwischen den ursprünglich lateinischen Auctortexten und der althochdeutschen Bearbeitung zu vermitteln. Die polyfunktionalen Formeln lassen sich unter Berücksichtigung ihres Funktionsspektrums auf einige dominante pragmatische Funktionen beschränken: Eine Gruppe von Routineformeln hat die Funktion, die Aufmerksamkeit der Leser/Hörer auf einen neuen Inhalt zu lenken, wodurch gleichzeitig auch Neues eingeführt und die Textstruktur gegliedert wird. Bei einer weiteren Gruppe steht die belehrende Funktion im Vordergrund, sie soll Wichtiges und Wissenswertes hervorheben. Bei einer dritten Gruppe ist der hinweisende Charakter der Formeln dominant. Sie haben zwar auch aufmerksamkeitssteuernde und belehrende Funktion, wollen aber in erster Linie auf Bemerkenswertes verweisen. Eine vierte Gruppe hat vornehmlich die Funktion, Vorausgegangenes auszudeuten, zu erklären oder zu übersetzen. Die belehrende Funktion tritt bei dieser vierten Gruppe gegenüber der erklärenden, veranschaulichenden deutlich in den Hintergrund, während bei den ersten drei Gruppen eine eindeutig differenzierende Zuordnung Schwierigkeiten bereitet. 
Hier wirkt sich die Polyfunktionalität der Formeln stärker aus, eine angemessene Beschreibung dieser Formeln erfordert daher die Berücksichtigung eines Funktionsspektrums.

Bislang ist dieser insgesamt sehr ergiebige Bereich von der phraseologischen Forschung jedoch wenig beachtet worden. Innerhalb der Nachwuchsforschergruppe „Historische Formelhafte Sprache und Traditionen des Formulierens (HiFoS)" soll diese Forschungslücke quellennah, kontextbezogen und systematisch geschlossen werden.

Universität Trier

\section{Anmerkungen}

Kontaktadresse: filatkin@uni-trier.de

1. Der Begriff Althochdeutsch bezieht sich auf den dialektalen Raum des Hochdeutschen in der Zeit zwischen ca. 750 bis ca. 1050 und umfasst den ältesten Überlieferungsstand des Hochdeutschen.

2. Das Projekt wird dort seit Juli 2007 im Fachteil Ältere deutsche Philologie (Wissenschaftliche Gastgeberin: Prof. Dr. Claudine Moulin) und im Historisch-Kulturwissenschaftlichen Forschungszentrum (HKFZ) Trier (http://www.hkfz.uni-trier.de) mit finanzieller Unterstützung der Alexander von Humboldt-Stiftung (Sofja Kovalevskaja-Preis 2006; Stifter: Bundesministerium für Bildung und Forschung) durchgeführt. Vgl. weitere Informationen zum Projekt unter: http://www.hifos.uni-trier.de.

3. Vgl. den Überblick in Filatkina (2009: 76-77).

4. Einen aktuellen Überblick über die relevante Forschungsliteratur bietet Lüger (2007: 445-447).

5. Diese vorläufigen Ergebnisse basieren auf den systematischen Auswertungen der althochdeutschen Segenssprüche in der HiFoS-Nachwuchsforschergruppe, die demnächst veröffentlicht werden sollen.

6. Wenngleich die Klosterkultur diese Funktionserweiterung erst ab dem 8./9. Jahrhundert erfährt (Haubrichs 1995: 53).

7. Die von Karl dem Großen initiierte Bildungsoffensive hatte dabei auch zum Ziel, Bildung und Wissenschaft als Voraussetzungen für die Übernahme politischer Aufgaben im Reich zu funktionalisieren (Goetz 2003: 253).

8. Vgl. dazu grundlegend Glauche (1970).

9. Einen konzentrierten Überblick über Bildung und Wissenschaft im Frühmittelalter bietet Hans-Werner Goetz (2003) in seiner Monographie Europa im frühen Mittelalter 500-1050 auf den Seiten 250-260.

10. Grotans (1998: 38) führt hierzu aus: "The revolutionary aspect of Notker's work is not so much that he used the vernacular - interlinear vernacular glosses were already 
old hat by the year 1000 - but the way in which he structured his classroom texts and the extent to which he wove the vernacular into the Latin commentative discourse."

11. Die spärliche Überlieferung volkssprachiger Texte zwischen dem 10. und 11. Jahrhundert lässt den Schluss zu, dass eine gewollte Abkehr von der volkssprachigen Schriftlichkeit vollzogen wurde. Vgl. hierzu auch de Boor (1979: 79).

12. Die Belege sowie ihre Übersetzungen sind der HiFoS-Datenbank (http://www.hifos. uni-trier.de) entnommen. Die jeweilige Beleg-ID findet sich in Klammern nach der Belegstelle. Die einzelnen Belege sind nach der Handschrift bzw. nach einer rezenten diplomatischen Edition erfasst. Abbreviaturen, die in den Handschriftentexten vorhanden waren, wurden aufgelöst und durch Klammern markiert. Die jeweilige Routineformel ist im Belegkontext unterstrichen.

13. Gesicherte Angaben lassen sich noch nicht machen, da das Korpus des Althochdeutschen gegenwärtig noch in Bearbeitung ist.

14. Überblickartig fasst Zerfaß (1995: 62-77) die Entwicklung und Tendenzen der Hoheliedexegese im Mittelalter zusammen. Grundlegend hierzu sind die Hoheliedstudien Ohlys (1958).

\section{Literatur}

\section{Primärliteratur}

Notker der Deutsche. 1995. De Interpretatione. Boethius' Bearbeitung von Aristoteles' Schrift Peri Hermeneias: Konkordanzen, Wortlisten und Abdruck des Textes nach dem Codex Sangallensis 818. Evelyn Scherabon Firchow (Hrsg.). Berlin: de Gruyter.

Notker der Deutsche. 1995. Categoriae. Boethius' Bearbeitung von Aristoteles' Schrift ,kategoriai'. Konkordanzen, Wortlisten und Abdruck der Texte nach den Codices Sangallensis 818 und 825. Evelyn Scherabon Firchow (Hrsg.), unter Mitarbeit von Richard Hotchkiss. Berlin: de Gruyter.

Notker der Deutsche. Martianus Capella, de Nuptiis Philologiae et Mercurii. Cod. Sang. 872 der Stiftsbibliothek St. Gallen.

Notker der Deutsche. Psalmenauslegung. Cod. 21 der Stiftsbibliothek St. Gallen.

Otfrid von Weißenburg : Evangelienbuch. Edition nach dem Wiener Codex 2687. Bd. I, Teil 1: Text. Wolfgang Kleiber (Hrsg.). Tübingen: Niemeyer.

Williram von Ebersberg. 2001. Expositio in Cantica Canticorum. Die älteste Überlieferung von Willirams Kommentar des Hohen Liedes. Edition, Übersetzung, Glossar. Rudolf Schützeichel und Birgit Meinecke (Hrsg.). Göttingen: Vandenhoeck \& Ruprecht.

\section{Sekundärliteratur}

Álvarez de la Granja, María (ed.). 2008. Fixed expressions in cross-linguistic perspective: A multilingual and multidisciplinary approach. Hamburg: Kovac.

Baldzuhn, Michael. 2002. Schulunterricht und Verschriftlichungsprozess: Forschungsansätze und Forschungsergebnisse. In Christel Meier et al. (eds.), Pragmatische 
Dimensionen mittelalterlicher Schriftkultur (Akten des Internationalen Kolloquiums, 26.-29. Mai 1999), 161-175. Munich: Wilhelm Fink.

Büge, Oskar. 1908. Die Beteuerungsformeln in Otfrids Evangelienbuch. Greifswald: Greifswald University dissertation.

Burger, Harald. 1977. Probleme einer historischen Phraseologie des Deutschen.PBB 99. 1-24.

Burger, Harald. 1983. Neue Aspekte der Semantik und Pragmatik phraseologischer Wortverbindungen. In Josip Matešič (ed.), Phraseologie und ihre Aufgaben: Beiträge zum 1. Internationalen Phraseologie-Symposium vom 12. bis 14. Oktober 1981 in Mannheim. Band 3, 24-34. Heidelberg: Groos.

Burger, Harald. 1998. Problembereiche einer historischen Phraseologie. In Wolfgang Eismann (ed.), Europhras 95. Europäische Phraseologie im Vergleich: Gemeinsames Erbe und kulturelle Vielfalt (Studien zur Phraseologie und Parömiologie 15), 79-108. Graz \& Leibnitz: N. Brockmeyer.

Burger, Harald. 2007. Phraseologie: Eine Einführung am Beispiel des Deutschen. 3rd edn. Berlin: Schmidt.

Burger, Harald, Annelies Buhofer \& Ambros Sialm. 1982. Handbuch der Phraseologie. Berlin: de Gruyter.

Coulmas, Florian. 1981. Routine im Gespräch: Zur pragmatischen Fundierung der Idiomatik. Wiesbaden: Akademische Verlagsgesellschaft Athenaion.

de Boor, Helmut. 1979. Die deutsche Literatur: Von Karl dem Großen bis zum Beginn der höfischen Dichtung, 770-1170. Band I. Munich: Beck.

Eikelmann, Manfred. 1999. Altsprochen wort: Sentenz und Sprichwort im Kontext der mittelalterlichen Gnomik. In Sieglinde Hartmann \& Ulrich Müller (eds.), Jahrbuch der Oswald von Wolkenstein Gesellschaft. Band 11, 299-315. Frankfurt/Main: Dr. Ludwig Reichert Verlag.

Eikelmann, Manfred. 2002. Zur historischen Pragmatik des Sprichworts im Mittelalter. In Dietrich Hartmann \& Jan Wirrer (eds.), Wer a sägt, muss auch B sägen: Beiträge zur Phraseologie und Sprichwortforschung aus dem Westfälischen Arbeitskreis, 95-105. Baltmannsweiler: Schneider Verlag Hohengehren.

Feilke, Helmuth. 1994. Common-sense-Kompetenz: Überlegungen zu einer Theorie des ,,sympathischen “ und ,,natürlichen “ Meinens und Verstehens. Frankfurt/Main: Suhrkamp.

Fellbaum, Christiane. 2007. Idioms and collocations: Corpus-based linguistic and lexicographic studies. London: Continuum.

Filatkina, Natalia. 2007. Pragmatische Beschreibungsansätze. In Harald Burger, Dmitrij Dobrovol'skij, Peter Kühn \& Neal R. Norrick (eds.), Phraseologie: Ein internationales Handbuch der zeitgenössischen Forschung. An International Handbook of Contemporary Research. Band 1 (Handbücher zur Sprach- und Kommunikationswissenschaft / Handbooks of Linguistics and Communication Science [HSK] 28), 132-158. Berlin \& New York: de Gruyter.

Filatkina, Natalia. 2009. Historische Formelhafte Sprache als „harte Nuss“ der Korpusund Computerlinguistik: Ihre Annotation und Analyse im HiFoS-Projekt. linguistikonline 39. http://www.linguistik-online.de/ (accessed 9 December 2009). 
Filatkina, Natalia. 2009. Und es duencket einem noch/wann man euch ansiehet / daß ihr Sand in den Augen habt. Phraseologismen in ausgewählten historischen Grammatiken des Deutschen. In Csaba Földes (ed.), Europhras 2006: Phraseologie disziplinär und interdisziplinär.

Filatkina, Natalia, Johannes Gottwald, Monika Hanauska \& Carolin Rößger. 2009. Formelhafte Sprache im schulischen Unterricht im Frühen Mittelalter: Am Beispiel der sogenannten ,Sprichwörter' in den Schriften Notkers des Deutschen von St. Gallen. Sprachwissenschaft 34(4). 341-397.

Friedrich, Jesko. 2006. Phraseologisches Wörterbuch des Mittelhochdeutschen: Redensarten, Sprichwörter und andere feste Wortverbindungen in Texten von 1050-1350. Tübingen: Niemeyer.

Friedrich, Jesko. 2007. Historische Phraseologie des Deutschen. In Harald Burger, Dmitrij Dobrovol'skij, Peter Kühn \& Neal R. Norrick (eds.), Phraseologie/Phraseology: Ein internationales Handbuch der zeitgenössischen Forschung: An International Handbook of Contemporary Research. Band 2 (Handbücher zur Sprach- und Kommunikationswissenschaft / Handbooks of Linguistics and Communication Science [HSK] 28), 1092-1106. Berlin \& New York: de Gruyter.

Glauche, Günther. 1970. Schullektüre im Mittelalter: Entstehung und Wandlungen des Lektürekanons bis 1200 nach den Quellen dargestellt (Münchener Beiträge zur Mediävistik und Renaissance-Forschung 5). Munich: Olms.

Goetz, Hans-Werner. 2003. Europa im frühen Mittelalter 500-1050. Stuttgart: Ulmer.

Grabmeyer, Bernhard. 1976. Die Mischsprache in Willirams Paraphrase des Hohen Liedes (Göppinger Arbeiten zur Germanistik 179). Göppingen: Kümmerle.

Green, Dennis. 1987. Zur primären Rezeption von Otfrids Evangelienbuch. In Rolf Bergmann (ed.), Althochdeutsch, Vol. 1, 737-771. Heidelberg: Winter.

Grotans, Anne. 1998. Simplifying Latin in Notkers classroom: Tradition and innovation. Journal of Germanic Language and Literature 10(1). 1-43.

Gülich, Elisabeth. 1997. Routineformeln und Formulierungsroutinen. In Rainer Wimmer \& F.-J. Berens (eds.), Wortbildung und Phraseologie (Studien zur deutschen Sprache 9), 131-175. Tübingen: Narr Francke Attempto.

Gülich, Elisabeth \& E. Henke. 1979/1980. Sprachliche Routine in der Alltagskommunikation. Die Neueren Sprachen 78. 513-530; 79. 2-33.

Haubrichs, Wolfgang. 1995. Geschichte der deutschen Literatur von den Anfängen bis zum Beginn der Neuzeit. Band. I/1: Die Anfänge: Versuche volkssprachiger Schriftlichkeit im frühen Mittelalter, 2nd edn. Tübingen: Niemeyer.

Haubrichs, Wolfgang \& Max Pfister. 1990. In Francia fui. Studien zu den germanischromanischen Interferenzen und zur Grundsprache der althochdeutschen „Pariser “ (Altdeutschen) Gespräche. Stuttgart: Steiner Franz.

Hellgardt, Ernst. 1979. Notkers des Deutschen Brief an Bischof Hugo von Sitten. In Klaus Grubmüller \& Ernst Hellgardt (eds.), Befund und Deutung: Zum Verhältnis von Empirie und Interpretation in Sprach- und Literaturwissenschaft. Festschrift für Hans Fromm, 169-192. Tübingen: Niemeyer.

Hellgardt, Ernst. 1981. Die exegetischen Quellen von Otfrids Evangelienbuch (Hermaea 41). Tübingen: Niemeyer. 
Kahl, Hans-Dietrich. 2000. ,Mission, II. Missionsgeschichte'. In Lexikon des Mittelalters CD-Rom-Ausgabe, Vol. 7, 669-670. Stuttgart.

Kühn, Peter. 1984. Pragmatische und lexikographische Beschreibung phraseologischer Einheiten: Phraseologismen und Routineformeln. In H.E. Wiegand (ed.), Studien zur neuhochdeutschen Lexikographie IV, 175-235. Hildesheim: Olms.

Linke, Angelika. 1996. Sprachkultur und Bürgertum: Zur Mentalitätsgeschichte des 19. Jahrhunderts. Stuttgart: Metzler.

Lorenz-Bourjot, Martine \& Heinz-Helmut Lüger. 2001. Phraseologie und Phraseodidaktik. Vienna: Praesens.

Lüger, Heinz-Helmut. 2007. Pragmatische Phraseme: Routineformeln. In Harald Burger, Dmitrij Dobrovol'skij, Peter Kühn \& Neal R. Norrick (eds.), Phraseologie: Ein internationales Handbuch der zeitgenössischen Forschung. An International Handbook of Contemporary Research. Band 1 (Handbücher zur Sprach- und Kommunikationswissenschaft / Handbooks of Linguistics and Communication Science [HSK] 28), 444-459. Berlin \& New York: de Gruyter.

Ochsenbein, Peter. 1991. L'enseignement et l'étude. In Werner Vogler (ed.), L'Abbaye de Saint-Gall. Rayonnement spirituel et culturel, 133-144. St. Gallen: Stiftsarchiv St. Gallen.

Ohly, Friedrich. 1958. Hohelied-Studien: Grundzüge einer Geschichte der Hoheliedauslegung des Abendlandes bis um 1200. Wiesbaden: F. Steiner.

Penzl, Herbert. 1984. Gimer min ros. German Quarterly 57. 392-401.

Riecke, Jörg. 2004. Die Frühgeschichte der medizinischen Fachsprache im Deutschen. Band 1: Untersuchungen. Berlin: de Gruyter.

Schröder, W. 1981. Glossae Cassellanae. In Kurt Ruh (ed.), Die deutsche Literatur des Mittelalters - Verfasserlexikon. Band 3, 2nd rev. edn., 61-63. Berlin: de Gruyter.

Schulz, Monika. 2000. Magie und Wiederherstellung der Ordnung. Frankfurt/Main: Lang.

Schulz, Monika. 2003. Beschwörungen im Mittelalter: Einführung und Überblick. Heidelberg: Winter.

Sonderegger, Stefan. 1978. ,Altdeutsche Gespräche‘. In Kurt Ruh (ed.), Die deutsche Literatur des Mittelalters - Verfasserlexikon. Band 1, 2nd rev. edn., 284-285. Berlin: de Gruyter.

Stein, Stephan. 1995. Formelhafte Sprache. Untersuchungen zu ihren pragmatischen und kognitiven Funktionen im gegenwärtigen Deutsch. Frankfurt/Main: Lang.

Zerfaß, Christiane. 1995. Die Allegorese zwischen Latinität und Volkssprache. Willirams von Ebersberg „Expositio in cantica canticorum “. Göppingen: Kümmerle. 
